\title{
Microcontainers as an oral delivery system for spray dried cubosomes containing ovalbumin
}

Nielsen, Line Hagner; Rades, Thomas; Boyd, Ben; Boisen, Anja

Published in:

European Journal of Pharmaceutics and Biopharmaceutics

Link to article, DOI:

10.1016/j.ejpb.2016.12.008

Publication date:

2017

Document Version

Peer reviewed version

Link back to DTU Orbit

Citation (APA):

Nielsen, L. H., Rades, T., Boyd, B., \& Boisen, A. (2017). Microcontainers as an oral delivery system for spray dried cubosomes containing ovalbumin. European Journal of Pharmaceutics and Biopharmaceutics, 118, 13-20. https://doi.org/10.1016/j.ejpb.2016.12.008

\section{General rights}

Copyright and moral rights for the publications made accessible in the public portal are retained by the authors and/or other copyright owners and it is a condition of accessing publications that users recognise and abide by the legal requirements associated with these rights.

- Users may download and print one copy of any publication from the public portal for the purpose of private study or research.

- You may not further distribute the material or use it for any profit-making activity or commercial gain

- You may freely distribute the URL identifying the publication in the public portal 
1 Microcontainers as an oral delivery system for spray dried cubosomes containing ovalbumin

2 Line Hagner Nielsen ${ }^{1, A}$, Thomas Rades ${ }^{2}$, Ben Boyd ${ }^{3}$, Anja Boisen ${ }^{1}$

$4 \quad{ }^{1}$ Department of Micro and Nanotechnology, Technical University of Denmark, Kgs. Lyngby, Denmark

$5{ }^{2}$ Department of Pharmacy, Faculty of Health and Medical Sciences, University of Copenhagen, Copenhagen,

6 Denmark

$7 \quad{ }^{3}$ Monash Institute of Pharmaceutical Sciences, Monash University, Melbourne, Australia

$9 \quad{ }^{A}$ Corresponding author: Technical University of Denmark, DTU Nanotech, Building 345C, Kongens 10 Lyngby, Denmark. Tel: +4545256843 Fax: + 4545887762 E-mail address: lihan@ nanotech.dtu.dk (L. 11 Hagner Nielsen). 


\section{Abstract}

The purpose of this study was to prepare cubosomes encapsulating the model antigen ovalbumin (OVA) via spray drying, and to characterise such cubosomes with a view for their potential application in oral vaccine delivery. Furthermore the cubosome formulation was loaded into polymeric microcontainers intended as an oral drug delivery system. The cubosomes consisted of commercial glyceryl monooleate, $\operatorname{Dimodan}^{\circledR}$, containing OVA and were surrounded with a dextran shell prepared by spray drying. Cryo-TEM was used to confirm that cubosomes were formed after hydration of the spray dried precursor powder. The precursor powder had a mean particle size of $1.3 \pm 0.1 \mu \mathrm{m}$, whereas the mean diameter of the dispersed cubosomes was $282 \pm 7 \mathrm{~nm}$ (PDI: 0.18 ) measured by dynamic light scattering. $8.5 \pm 0.3 \%(\mathrm{w} / \mathrm{w})$ of OVA was present in the cubosome powder and OVA was found released slowly over the first $70 \mathrm{~h}$, followed by a more rapid release. Total release of $47.9 \pm 2.8 \%$ of loaded OVA occurred over $96 \mathrm{~h}$ in a buffer at $\mathrm{pH} 6.8$. When the powder was filled into microcontainers, and the opening covered with the $\mathrm{pH}$ sensitive polymer Eudragit S100, the $\mathrm{pH}$ sensitive 'lid' was intact at gastric $\mathrm{pH}$, but release of OVA from the cubosomes and microcontainers occurred at $\mathrm{pH} 6.8$, releasing $44.1 \pm 5.6 \%$ of the OVA in $96 \mathrm{~h}$. Small-angle X-ray scattering (SAXS) revealed that the 'dry' particles possessed an internal ordered lipid structure (lamellar and inverse micellar phase) by virtue of a small amount of residual water, and after hydration in buffer at $\mathrm{pH} 6.8$, the particles formed the hexagonal inverse cubic phases, thereby indicating that cubosomes were formed when released from microcontainers. 


\section{Introduction}

Vaccination is often regarded as the most significant contribution to public health and disease prevention and moreover, it is a very cost-effective medical intervention [1,2]. Vaccination has reduced the morbidity and mortality resulting from diseases such as tuberculosis and smallpox and has thereby saved millions of lives. In spite of this, many infectious diseases remain endemic in large parts of the world, and therefore vaccination is an area in continuous development [1,2].

Most vaccines are administered by injection and there are only a few oral vaccines on the market such as rotavirus vaccine (as solution or suspension) and a capsule with vaccine formulation against typhoid fever [3]. Although, the oral route can be beneficial for vaccine administration [4,5]. Some of the advantages of oral vaccines are the ease of administration and an increased safety compared to injections. In addition, there is also a great potential for mass vaccination without the requirements of trained personnel $[4,6]$. Furthermore, oral vaccines have the ability to induce both mucosal and systemic immune responses [6,7], as shown in the 1990s with several HIV vaccines [8], and they are therefore considered ideal for combating infectious diseases. Although, oral vaccines have several attractive features, there are some major challenges.

The target of vaccine formulations in the gastro-intestinal (GI) tract is the M-cells in the intestine [9]. The antigen might be damaged, when passing through the harsh environment of the Gl tract, which in turn will lead to the need for large doses. In addition, there is a poor transport of the antigen across the intestinal epithelium [4].

Traditional vaccines are mainly composed of heat-inactivated bacteria or viruses resulting in high immunogenicity. The risk with these types of vaccines is that they, in the body, can change to the active state and thereby infect the patients with the bacteria or virus and thus, leading to unwanted side effects $[1,10]$. Consequently, new generation vaccines are developed with subunit antigens. These subunit antigens are highly purified components of pathogens and thereby chemically well-defined. Hence, there is a much higher safety than for traditional antigens, but as the subunit antigens lack most of the features of the original pathogen they tend to be poorly immunogenic $[1,10]$. Therefore, to succeed with oral vaccine delivery, delivery systems need to be developed, in which the antigen can be encapsulated into particles $[11,12]$. These particles will assure presentation of the antigen to the antigen-presenting cells, but can also stabilise and release the antigen over an extended period of time [10]. Some particles will provide an adjuvant effect in themselves, but potent adjuvants can in addition also be added to the particulates for inducing an effective immunity [13].

There are many possibilities for vaccine delivery systems, and some of the most common ones are: polymeric micro- and nanoparticles, immunostimulatory complexes and liposomes [14,15]. Cubosomes have also shown to be an efficient delivery system for vaccines [11]. Cubosomes contain a highly twisted, continuous lipid bilayer with two congruent, non-intersecting water channels, giving the particles both hydrophobic and hydrophilic domains [11]. This offers great flexibility with respect to the types of compounds, that can be incorporated into the particles [16,17]. Rizwan et al. found that significantly higher amounts of antigen can be encapsulated in cubosomes compared to liposomes due to the larger surface area of cubosomes, and moreover in cubosomes, the antigen was also retained more efficiently compared to liposomes [11]. 
Traditionally, cubosomes are produced by mixing monoolein or phytantriol and water and thereby creating a high-energy dispersion followed by colloidal stabilisation using polymeric stabilisers $[11,18,19]$. However, it can be desirable to have the vaccine particles in a powder form (here termed "precursors"), and precursors of cubosomes have earlier been produced by either freeze drying [20] or spray drying [21-23]. In the spray drying process, dry powder precursors have the active ingredient incorporated, and upon hydration colloidally stable cubosomes are spontaneously formed. The powder form of the vaccine formulation can be advantageous in terms of stability of the antigen. Also, there is no need for a cold-chain storage which is needed for traditional vaccines [4].

After oral administration of the vaccine formulations, the antigen needs to be protected in the stomach and during transportation to the small intestine. In the small intestine, the vaccine particles should be delivered to the microfold $(\mathrm{M})$ cells of the peyer's patches as they will present the antigen to the underlying immune cells and thereby obtain an immune response [24]. The particles, carrying the antigen (and adjuvant), can give some protection of the antigen through the Gl environment, but often the particles will also degrade on the way to the intestine, and therefore more advanced drug delivery systems can be necessary. An example of these advanced drug delivery systems is microcontainers. Microcontainers are polymeric, cylindrical devices in the micrometre size range (Fig. 1) [25-27]. They have the potential for targeted and/or sustained delivery in the Gl tract [28]. Some of the advantages of the microcontainers are that size and shape can be controlled very precisely. Furthermore, the devices allow for unidirectional release, as only one side of the microcontainer is open, compared to more conventional microparticles where release can occur from the whole surface area. This has shown to increase the drug concentration at the microdevice-cell interface and thereby, allowing for increased permeation of the drug in vitro leading to enlarged oral bioavailability of the drug $[27,29,30]$. In addition, the antigen can be protected inside the cavity of the microcontainer from the harsh environment of the stomach until release is desirable [31,32]. The microcontainers have previously shown to interact with the intestinal mucus resulting in prolonged drug absorption [27]. It is reported in the literature that one way to improve oral vaccine delivery is to extend the intestinal residence time [13], hence, the microcontainers can be a promising platform for this purpose. In this paper, SU-8 (an epoxy photoresist) was used as a model polymer for fabrication of the microcontainers $[25,26,31]$, but microcontainers have also been fabricated using biopolymers such as polyL-lactic acid (PLLA) [33,34].

The aim of this study was, as a proof-of-concept, to prepare and characterise cubosomes loaded with ovalbumin (OVA) in a spray dried powder form for future application in oral vaccine delivery. The precursor powder was filled into microcontainers for protection and release control, and the in vitro release was studied together with small-angle X-ray scattering (SAXS) to confirm whether cubosomes were released from the microcontainers as internally structured particles.

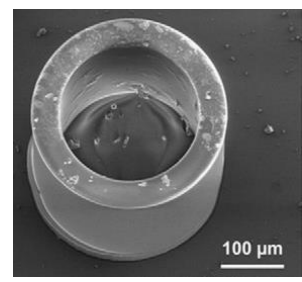

Fig. 1: SEM image of an SU-8 microcontainer with an inner diameter of $223 \mu \mathrm{m}$ [26]. 


\section{Materials and methods}

\section{Materials}

OVA was purchased from TCl Europe (Zwijndrecht, Belgium). Dimodan ${ }^{\circledR}$ D90 was kindly donated by Danisco (Grindsted, Denmark). Dextran (from Leuconostoc Mesenteroides) and potassium dihydrogen phosphate were acquired from Sigma-Aldrich (St. Louis, MO, USA). Pierce BCA Protein Assay kit was purchased from Thermo Fisher Scientific (Rockford, IL, USA). Deionised water was obtained from an SG Ultra Clear water system (SG Water USA, LLC, Nashua, NH, USA) and was freshly produced in all cases. All other chemicals used were of analytical grade.

\section{Spray drying of precursors for cubosomes containing OVA}

Cubosomes were prepared using a commercial source of glyceryl monooleate (GMO), Dimodan ${ }^{\circledR}$ MO 90/D. The cubosomes were loaded with OVA as a model antigen, and the particles were surrounded by a dextran

127 shell. The particles were prepared by first dissolving GMO in ethanol $(1.78 \mathrm{w} / \mathrm{v} \%)$, and then mixing with a solution of OVA in MilliQ water ( 0.075 final w/w \% of OVA). After $1 \mathrm{~h}$ of mixing, dextran dissolved in MilliQ water $(1.77 \mathrm{w} / \mathrm{v} \%)$ was added to the GMO/OVA solution $(0.72 \mathrm{w} / \mathrm{w} \%$ of GMO + OVA), and the final solution was spray dried using a B 290 Büchi mini spray dryer (Büchi Labortechnik AG, Flawil, Switzerland). Free OVA was not removed prior to the spray drying process.

For the spray drying of the precursors, a $0.7-\mathrm{mm}$ nozzle was used and air was utilised as the drying medium. Spray drying was performed at an inlet temperature of $200{ }^{\circ} \mathrm{C}$ resulting in an outlet temperature of approximately $85{ }^{\circ} \mathrm{C}$. The drying flow rate was set to $32 \mathrm{~m}^{3} / \mathrm{h}$ and an aspirator capacity of $80 \%$ with a feed rate of $4 \mathrm{~mL} / \mathrm{min}$ was used. Particles without OVA were also produced as blank particles and used as reference.

\section{Cryo-TEM of cubosomes}

The precursors for the cubosomes with OVA were dispersed in MilliQ water at a concentration of $1 \mathrm{mg} / \mathrm{mL}$. The samples for the Cryo-TEM studies were prepared in a controlled environment vitrification system (CEVS). A small amount of the sample $(5 \mu \mathrm{L})$ was put on a carbon film supported by a copper grid and blotted with filter paper to obtain a thin liquid film on the grid. The grid was quenched in liquid ethane at $-180{ }^{\circ} \mathrm{C}$ and transferred to liquid nitrogen $\left(-196^{\circ} \mathrm{C}\right)$. The samples were then examined using a Tecnai G2 F30 Transmission Electron Microscope (FEl, Eindhoven, The Netherlands) operating at a voltage of $300 \mathrm{kV}$ and a working temperature of $-180^{\circ} \mathrm{C}$. Images were recorded using Gatan UltraScan $1000(2 \mathrm{k} \times 2 \mathrm{k}) \mathrm{CCD}$ camera (Gatan, California, USA).

\section{Size of particles}

The size of the dry particles with the dextran shell was measured using aerosizer particle size analyser (Model 3321, TSI Incorporated, MN, USA) by setting the pump to 1.37 bar and with a capillary flow of 8 $\mathrm{L} / \mathrm{min}$. A small amount of powder was distributed on the plate and the particle size was measured in six replicates.

For the particles dispersed in water, the particle size distribution (Z-average), polydispersity (PDI) and zeta potential were determined using dynamic light scattering (Malvern Zetasizer, NanoZs ZEN 3600, Malvern, UK). Measurements were performed at $37^{\circ} \mathrm{C}$, and the results presented are the mean of three successive 
measurements of $100 \mathrm{~s}$ of at least three independent samples. Samples were diluted with water to adjust the signal level.

\section{OVA present in the cubosomes}

Precursor powder (10 mg) was added to a solution of $20 \mathrm{mM}$ phosphate buffer, pH 6.8 containing $5 \%$ Triton X-100. After vortex mixing, the cubosomes were dissolved and a sample of $200 \mu \mathrm{L}$ was taken out. A BCA Protein Assay kit was used to determine how much OVA was present in the cubosome powder by following the procedures for the standards and samples recommended by the manufacturer. The same process was performed with the blank cubosomes to check for any cross activity of the formulation. The absorbance was measured at $562 \mathrm{~nm}$ on a plate reader, and the obtained absorbance values were analysed against the standard curves prepared on the same day as the samples. OVA entrapment was then determined by calculating the difference between the total OVA added before the spray drying process and the free fraction of OVA in the solution. The experiments were performed in triplicates.

\section{Fabrication of SU-8 microcontainers}

Production of the microcontainers involved two steps of photolithography with the negative epoxy-based photoresist, SU-8 [26,32]. The microcontainers were structured on a fluorocarbon coating deposited on top of the supporting silicon wafer by plasma polymerisation. This enabled dry removal of the fabricated SU-8 devices from the support substrate in order to obtain individual microcontainers if needed $[27,35]$. The fabricated microcontainers had an inner diameter of $223 \pm 3 \mu \mathrm{m}$ and a height of $270 \pm 3 \mu \mathrm{m}$ (mean $\pm S D, n=6$ ). Silicon wafers supporting the microcontainers were finally cut into squares of $12.8 \times 12.8 \mathrm{~mm}^{2}$ using an Automatic Dicing Saw from DISCO (Kirchheim b. München, Germany). Each chip contained arrays of $25 \times 25$ containers with a pitch of $450 \mu \mathrm{m}$.

\section{Filling of microcontainers with powder precursors}

Powder precursors were manually distributed on the microcontainer chip. The excess drug in between the microcontainers was then removed with pressurised air, resulting in powder-filled microcontainers [27]. The chip with microcontainers was weighed before and after filling to determine the amount of drug filled into the microcontainers.

\section{Spray coating of the filled microcontainers with Eudragit S100}

A spray coating system (ExactaCoat, Sono Tek, USA) equipped with an ultrasonic nozzle actuated at $120 \mathrm{kHz}$ [36] was used to deposit Eudragit S100 (dissolved to a $2 \%(\mathrm{w} / \mathrm{w}$ ) solution in isopropyl alcohol) on the cavity of the drug-filled microcontainers in a set-up similar to previously described [33]. The generator power was set to $1.5 \mathrm{~W}$, and the polymer solution was pumped through the nozzle at a flow rate of $100 \mu \mathrm{L} / \mathrm{min}$. Nitrogen gas at a pressure of 10 mbar was used to direct the beam of droplets onto the microcontainers, and the distance between nozzle and substrate was $40 \mathrm{~mm}$ with the beam diameter on the substrate being approximately $4 \mathrm{~mm}$. The lateral movements of the nozzle were controlled by an $x-y$ stage and the nozzle path was defined in the equipment software. The nozzle was moved line-by-line at a speed of $25 \mathrm{~mm} / \mathrm{s}$, and the coating was repeated 60 times to obtain a coating thickness in the $\mu \mathrm{m}$ range.

Release of OVA from the cubosomes 
In vitro release of OVA from the cubosomes unconfined (bulk powder) and confined in microcontainers coated with Eudragit S100 was investigated on a $\mu$ DISS profiler ( $p$ ION INC, Woburn, MA). In both release studies, each channel was calibrated with its own OVA standard curve prior to the experiments. For the calibration curves, aliquots of OVA in water stock solution were repeatedly added to $10 \mathrm{~mL}$ of either a $\mathrm{HCl}$ solution or a phosphate buffer in order to achieve a range of defined standard concentrations, and the UV spectrum of each standard was recorded. The release experiments were performed at $37 \pm 0.5^{\circ} \mathrm{C}$ using a stirring rate of $200 \pm 5 \mathrm{rpm}$ using $20 \mathrm{~mm}$ path length in situ UV probes on a $\mu$ DISS profiler. The absorbance data was evaluated using $280 \mathrm{~nm}$ on the standard curve and utilising the $2^{\text {nd }}$ derivative function in the $\mathrm{Au}$ Pro software affiliated with the $\mu$ DISS profiler.

The release of OVA from the precursor powder was studied in $20 \mathrm{mM}$ phosphate buffer, $\mathrm{pH} 6.8$ for $96 \mathrm{~h}$. The in situ UV probes were situated in each sample vial containing $10 \mathrm{mg}$ of powder and $10 \mathrm{~mL}$ of phosphate buffer was added. The probes scanned and detected the absorbance of released OVA.

The release studies from the microcontainers were performed in a set-up similar to one previously described $[25,27,33]$. The chips with microcontainers were attached to cylindrical magnetic stirring bars (using carbon pads) and placed in the bottom of sample vials. The chips were covered with $10 \mathrm{~mL}$ of $0.1 \mathrm{M}$ $\mathrm{HCl} \mathrm{pH} 1.6$ for $2 \mathrm{~h}$ and subsequently, the medium was changed to $10 \mathrm{~mL}$ of $20 \mathrm{mM}$ phosphate buffer, $\mathrm{pH}$ 6.8 for $96 \mathrm{~h}$, and the in situ UV probes detected the absorbance.

Both sets of experiments were performed in 3 replicates.

\section{Scanning electron microscopy of the microcontainers}

SEM was utilised to examine the microcontainers after filling, after spray coating of the lid of Eudragit S100 , and after release in phosphate buffer at $\mathrm{pH}$ 6.8. The examinations were carried out using a Phenom Pro scanning electron microscope (Phenom World, Eindhoven, the Netherlands). Prior to the investigations, the microcontainer chip was mounted onto metal stubs, and imaging was performed at an operation voltage of $10 \mathrm{kV}$ with a $600 \mathrm{x}$ magnification.

\section{SAXS determination of the structure of cubosomes loaded into microcontainers}

The SAXS/WAXS beamline at the Australian Synchrotron, Clayton, Australia [37] was used to determine the internal structure of the spray dried particles, when the cubosomes were confined in microcontainers and released from the devices. The X-ray beam had an energy of $11 \mathrm{keV}$, and the 2D SAXS patterns were collected using a Pilatus $1 \mathrm{M}$ camera (active area $169 \times 179 \mathrm{~mm}^{2}$ with a pixel size of $172 \times 172 \mu \mathrm{m}$ ), which was located $900 \mathrm{~mm}$ from the sample position. The total q range for the instrument configuration outlined above was $0.02<q<1.06 \AA^{-1}$, and 2D SAXS patterns were collected for $1 \mathrm{sec}$. The in-house designed computer software 'ScatterBrain' was used to acquire and reduce these 2D patterns to $1 \mathrm{D}$ intensity versus q profiles. The powder-filled microcontainers were separated from the base using a scalpel, and filled into a $1.5 \mathrm{~mL}$ capillary and SAXS patterns were acquired in dry state followed by addition of $50 \mu \mathrm{L}$ of MilliQ water, where after patterns were acquired for a time period of $80 \mathrm{~min}$. The set-up with empty microcontainers as an example can be seen in Fig. 2A, with an image of the microcontainers in a capillary in the X-ray beam shown in Fig. 2B. 
A)

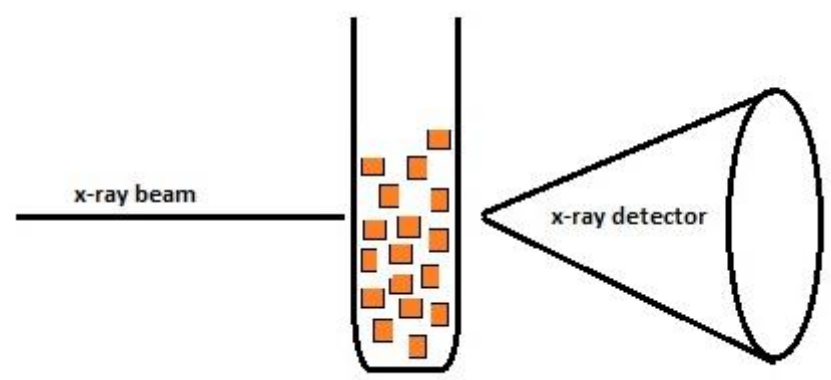

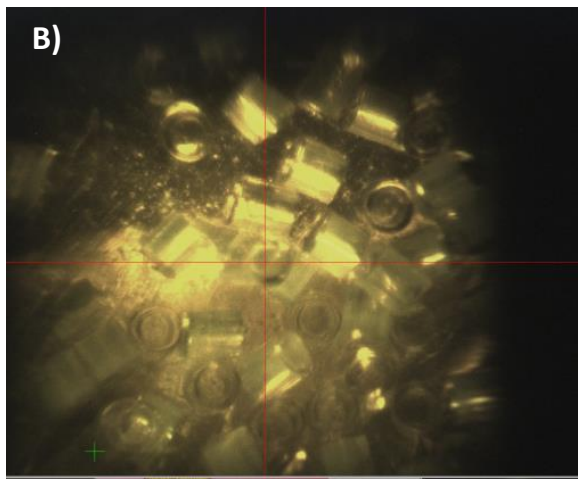

Fig. 2: A) Schematic of the SU-8 microcontainers filled into a capillary to be used in the SAXS/WAXS synchrotron. B) Micrograph showing the set-up with the microcontainers in the x-ray beam.

\section{$\underline{\text { Statistics }}$}

The data are expressed as mean \pm standard deviation (SD). Where appropriate, statistical analysis was carried out using Student t-tests using GraphPad Prism version 7.00 (GraphPad Software Inc., CA, USA). Pvalues below $5 \%(p<0.05)$ were considered statistically significant.

\section{Results and discussion}

For the production of the powder precursors of cubosomes, spray drying was chosen as this is a simple technique converting a solution to powder in a one-step process [38]. GMO has for many years been one of the lipids of choice for producing cubosomes, as it is non-toxic, biocompatible and biodegradable [39], and therefore it was decided to produce GMO particles in this study. The spray drying technique is convenient for producing the powder precursors, but GMO can be challenging to spray dry as it immediately forms the cubic phase upon hydration. Spicer et al. studied the effect of applying ethanol as a hydrotrope and this resulted in the formation of a low-viscous emulsion that was easily spray dried [21]. For this reason, in this study, GMO was first dissolved in ethanol and then added to the aqueous dextran solution. It has been reported that GMO itself produces sticky agglomerates after spray drying, and to obtain a more flowable powder an aqueous starch or a dextran solution can be added prior to spray drying resulting in the GMO being encapsulated in a dry starch or dextran shell [21-23]. In this work, it was chosen to add dextran as the anti-cohesion agent, and the produced powder was flowable and easy to hydrate. After production of the GMO powder precursors, the powder was hydrated and cryo-TEM was performed to identify whether cubosomes were obtained. It can be observed in Fig. 3 that cubic structures were found after hydration of the powder. 


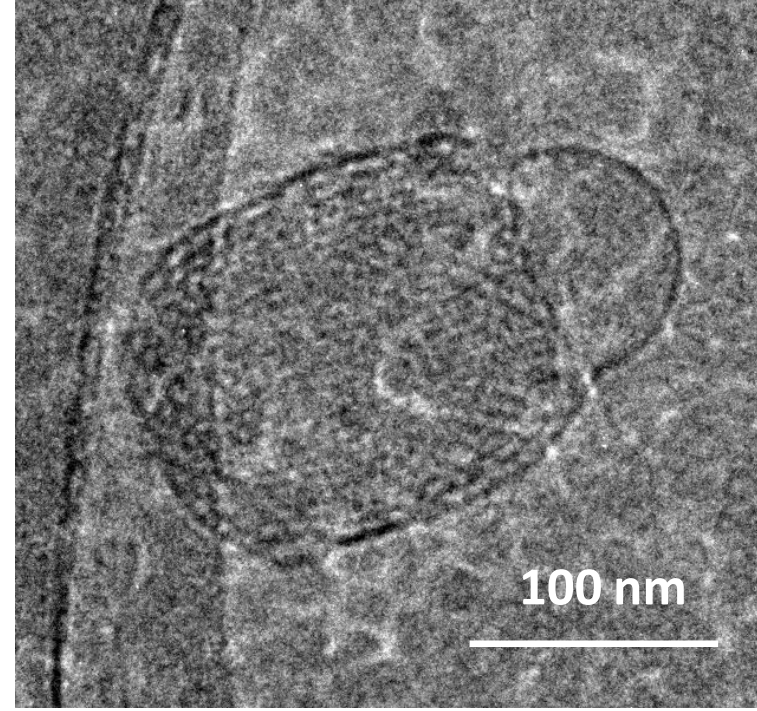

Fig. 3: Cryo-TEM image of a representative hydrated particle with a distinct cubic liquid crystalline structure. The resolution in cryo-TEM images is limited by the presence of dissolved dextran.

\section{In vitro characterisation of the particulates}

The size, shape and surface charge of a particulate vaccine carrier will influence its performance as a vaccine [40]. The dry powder with OVA and the dextran shell had a size of $1.3 \pm 0.1 \mu \mathrm{m}$, whereas the dry blank particles without OVA had a size of $1.6 \pm 0.1 \mu \mathrm{m}$. After hydration, self-assembled, close to neutrally charged nanoparticles were formed, with mean size of $146.1 \pm 1.3 \mathrm{~nm}$ and $281.7 \pm 7.4 \mathrm{~nm}$ for the blank and OVA-loaded particles, respectively (Table 1). There was a significant size difference between the blank and OVA-loaded particles ( $p$-value <0.0001), and the PDI for both formulations was low, indicating homogeneous formulations. The particles were much smaller than those reported by Spicer et al., where the dry particles had a diameter of $24 \mu \mathrm{m}$, and in the hydrated form the cubosomes were in average $0.6 \mu \mathrm{m}$ with a size distribution from 0.1 to $5 \mu \mathrm{m}$ [22]. In general, it is reported that the particle size should be between $20 \mathrm{~nm}$ to $10 \mu \mathrm{m}$ to be well recognised by the immune system [11], but more specifically for oral vaccine formulations, a size between $200-500 \mathrm{~nm}$ can be advantageous for uptake into the antigenpresenting cells after oral administration [40,41]. In relation to this, it can be observed that the size of the cubosomes with OVA is in this size range, and the cubosomes should therefore be able to be taken up by the antigen-presenting cells.

Table 1: Size measurements of the hydrated cubosomes with and without OVA dispersed in MilliQ water. The measurements were performed using dynamic light scattering in triplicates, and data are represented as mean $\pm \mathrm{SD}$

\begin{tabular}{|l|l|l|l|}
\hline & Z-average $(\mathbf{n m})$ & PDI & Zeta potential $(\mathbf{m V})$ \\
\hline Blank particles & $146.1 \pm 1.3$ & $0.15 \pm 0.02$ & $-0.43 \pm 0.077$ \\
\hline Particles with OVA & $281.7 \pm 7.4$ & $0.18 \pm 0.11$ & $-0.18 \pm 0.042$ \\
\hline
\end{tabular}

\section{Presence and release of OVA in and from the particles}

Before the release measurements, it was initially determined that $8.5 \pm 0.3 \%(w / w)$ OVA was present in the cubosome powder. It is also well-known that cubosomes often provide a sustained release of a drug [39], and this is also observed in this study, where release studies in buffer at $\mathrm{pH} 6.8$ showed that during the first 
$70 \mathrm{~h}$, OVA was slowly released, followed by a more rapid release from $70-80 \mathrm{~h}$. A total release of $47.9 \pm 2.8 \%$ was observed in relation to the total loading of OVA in the cubosomes over a $96 \mathrm{~h}$ period (Fig. 4). It can be seen that there is a significant burst release of OVA from the cubosomes (insert in Fig. 4) of $18.2 \pm 1.6 \%$ in the first $10 \mathrm{~min}$. This is probably caused by the release of OVA from the powder just when the powder precursors are dispersed in the aqueous solution, and thereafter the OVA entrapped in the channels of the cubosomes is released. In the literature, it is reported that OVA was released during $168 \mathrm{~h}$ from cubosomes resulting in a complete release [18]. A study preparing precursors of cubosomes by spray drying, but encapsulating the highly lipophilic drug, efavirenz, also is reporting on a burst release of the drug of up to $16 \%$, with a total release in $12 \mathrm{~h}$ of up to $56 \%$, again indicating that when dispersing powder precursors in aqueous solution a burst release is occurring [23]. A sustained release of OVA is also observed in this study and this could be beneficial when developing vaccine formulations [42].

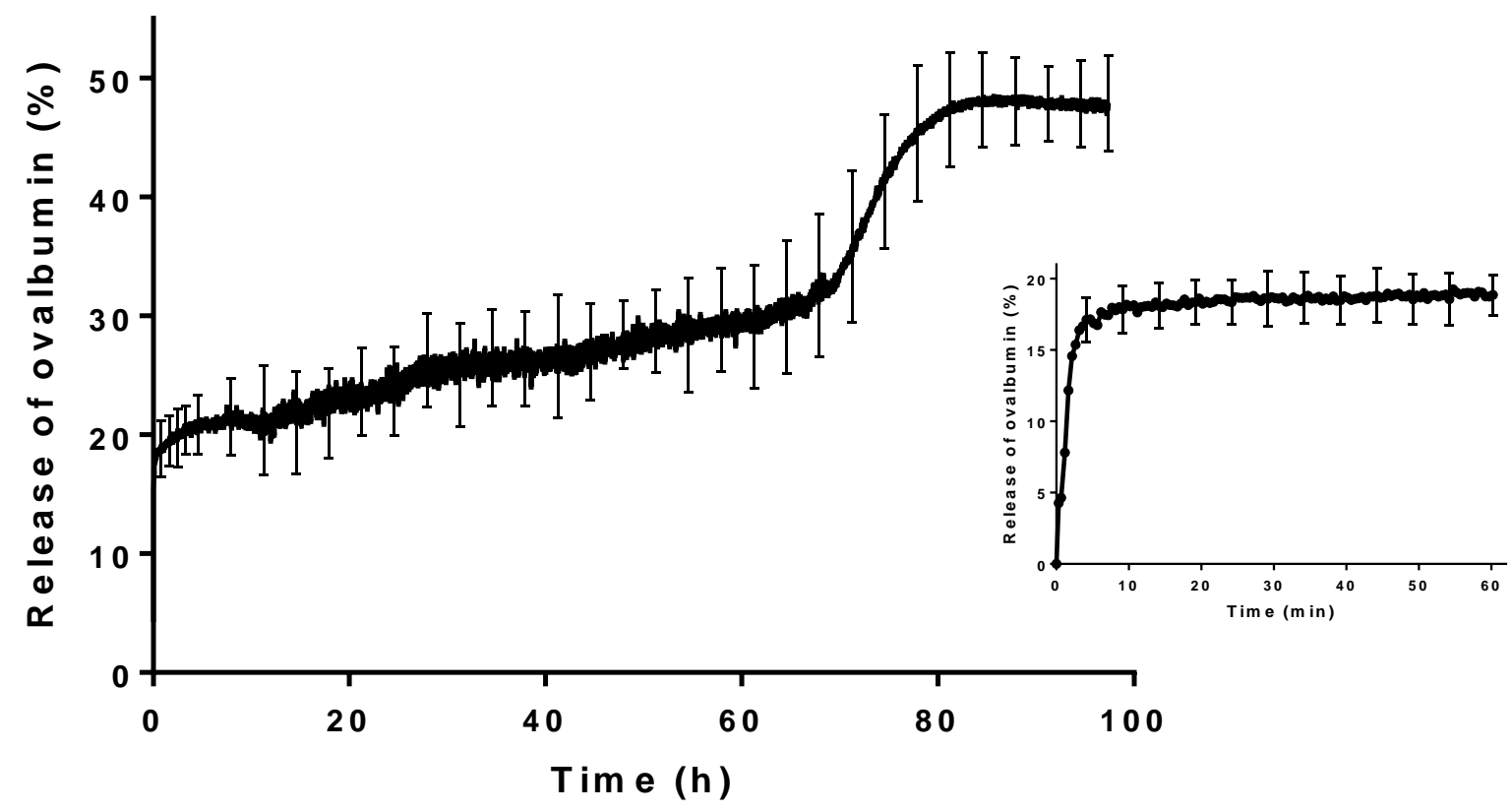

Fig. 4: Release of OVA from the cubosomes in $20 \mathrm{mM}$ phosphate buffer $\mathrm{pH} 6.8$, expressed as $\%$ of the total content of OVA. The insert is showing the release over the first $60 \mathrm{~min}$. The release study was performed in triplicates, and the data represent mean \pm SD.

Internal structure of particles formed upon hydration from microcontainers

SAXS/WAXS can be used to detect phase transformations in self-assembled lipid systems, and this was utilised to identify whether particles released from the microcontainers contained internal nanostructures consistent with cubosomes. Fig. 5 shows the plot of intensity versus the scattering vector q obtained from the release of GMO particles in dry form and when the microcontainers containing the particles were dispersed in water for a period of $80 \mathrm{~min}$. For the dry particles, it can be observed that there are three equally spaced peaks in the diffractogram (Fig. 5), indicating that the dry particles are in a lamellar phase with the lattice parameter of $49.5 \AA$ (Table 2). There is also an inverse micellar phase present, indicated by the broad peak at $q \sim 0,2 \AA^{-1}$ in the diffractogram) with a D-spacing of $31.1 \AA$. This can be explained by the 
presence of residual moisture in the spray dried powder. According to the phase diagram of GMO in water, the inverse micellar phase and lamellar phase coexist at approximately $5 \%$ water $[43,44]$, consistent with Spicer et al. reporting approximately $5 \%(w / w)$ of moisture content in their spray dried cubosome powders [22].

After hydration (here exemplified by the diffractogram at $50 \mathrm{~min}$ ), the liquid crystalline nanostructured particles showed a mix of phases, with peak indexing indicating coexisting inverse hexagonal $\left(\mathrm{H}_{2}\right)$ phase (peaks at $\sqrt{ } 1: \sqrt{ } 3: \sqrt{ } 4)$,. Pn $3 m$ cubic phase (peaks at $\sqrt{ } 2: \sqrt{ } 3: \sqrt{ } 4$ ), and la3d cubic phase (peaks at $\sqrt{ } 6: \sqrt{ } 8$ ). These three phases appear in the GMO + water phase diagram [44], and the calculated lattice parameters are listed in Table 2. The presence of $\mathrm{H}_{2}$ and $\mathrm{Pn3m}$ cubic phases for commercial GMO samples in water might be expected at full hydration, however the la3d cubic phase is only expected at less than full hydration o the lipid. Therefore it is proposed that the particles were not completely hydrated after $50 \mathrm{~min}$, which is also supported by the fact that after $80 \mathrm{~min}$ the la3d phase appears even less prominent.

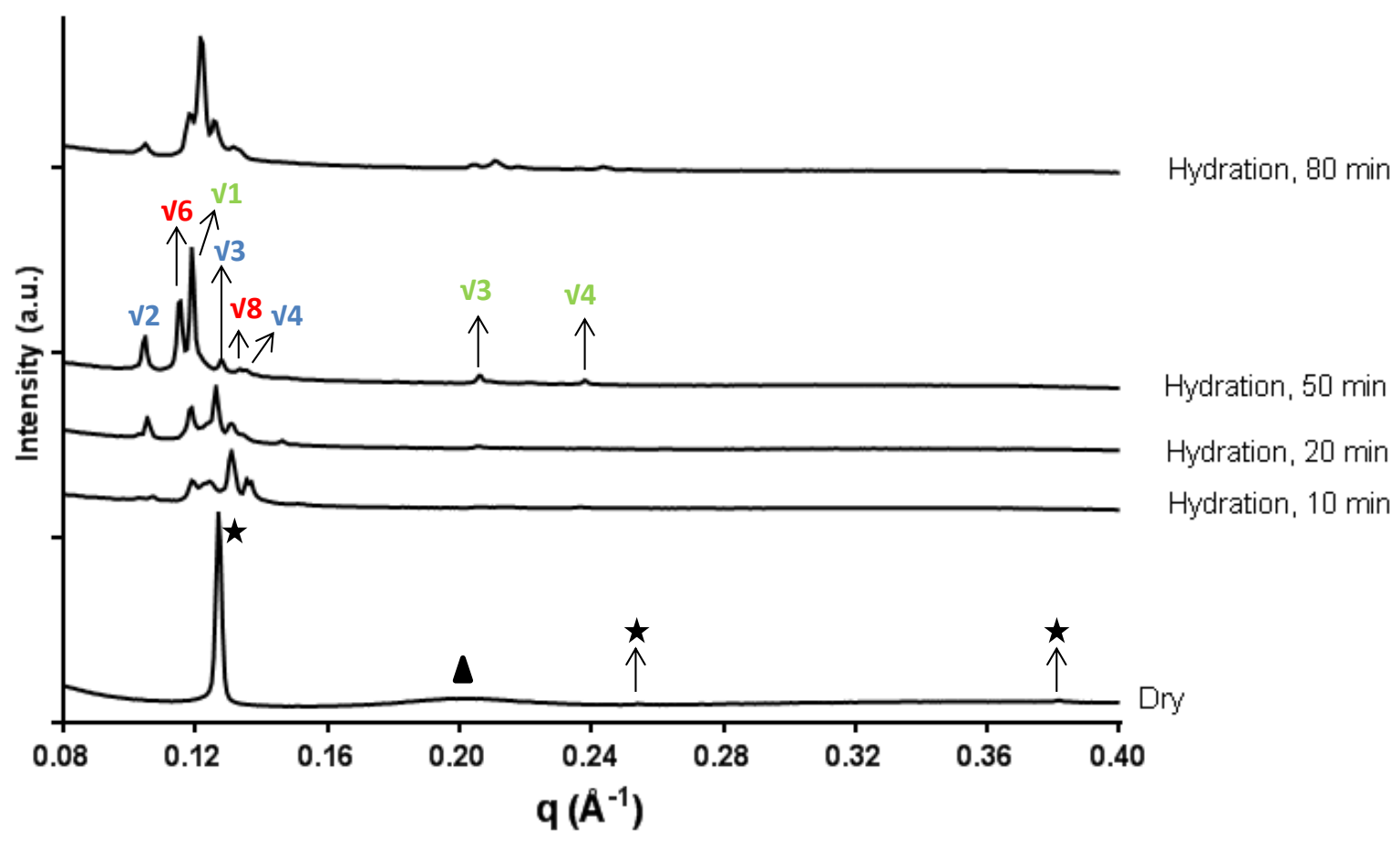

Fig. 5: 2D SAXS patterns were collected from cubosomes confined in microcontainers and followed while the cubosomes were released from the microcontainers in MilliQ water. The cubosome filled microcontainers were enclosed in a glass capillary during hydration for up to $80 \mathrm{~min}$. After $50 \mathrm{~min}$ of hydration the particles show a mix of phases with inverse hexagonal $\left(H_{2}\right)$ phase (peaks at $\left.\sqrt{ } 1: \sqrt{ } 3: \sqrt{ } 4\right)$, Pn $3 m$ cubic phase (peaks at $\sqrt{ } 2: \sqrt{ } 3: \sqrt{ } 4$ ), and la3d cubic phase (peaks at $\sqrt{ } 6: \sqrt{ } 8$ ).

Table 2: Phase structure and lattice parameters obtained from SAXS measurements of dry particles and particles released from microcontainers after hydration, here with an example after 50 min of hydration. 


\begin{tabular}{|l|l|l|}
\hline & Lattice parameters $(\AA)$ & \\
\hline & Dry particles & Hydration for $\mathbf{5 0}$ min \\
\hline Lamellar phase, $\mathbf{L}_{\alpha}$ & 49.5 & \\
\hline Inverse micellar phase, $\mathbf{L}_{\mathbf{2}}$ & 31.1 & 61.0 \\
\hline Inverse hexagonal phase, $\mathbf{H}_{\mathbf{2}}$ & & 84.6 \\
\hline $\begin{array}{l}\text { Inverse bicontinuous cubic } \\
\text { phase, Pn3m }\end{array}$ & & 132.7 \\
\hline $\begin{array}{l}\text { Inverse bicontinuous cubic } \\
\text { phase, la3d }\end{array}$ & & \\
\hline
\end{tabular}

Loading of precursors into the microcontainers and coating of the $\mathrm{pH}$ sensitive lid

After successfully loading the cubosomes into the microcontainers (Fig. 6A), the cavity of the microcontainers was coated with Eudragit S100 (Fig. 6B) as this polymer will dissolve at a pH value of approximately 7 corresponding to the $\mathrm{pH}$ found in the small intestine around the $\mathrm{M}$ cells [9].
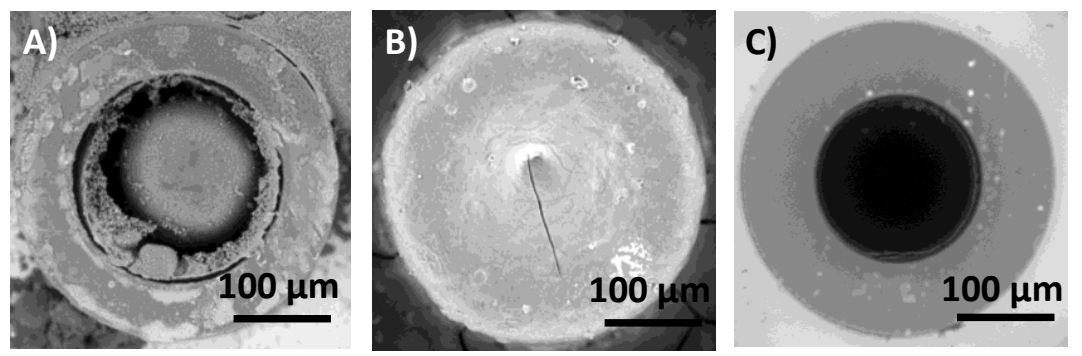

Fig. 6: SEM images of A) a cubosome-filled microcontainer, B) a filled microcontainers with a lid of Eudragit $\mathrm{S} 100$, and C) an empty microcontainer after release study in phosphate buffer $\mathrm{pH} 6.8$.

\section{Release of OVA loaded cubosomes from coated microcontainers}

The coating on the cavity of the microcontainers can prevent the release until the intestine [33], and a large dose (approximately $2 \mu \mathrm{g}$ of powder) of the vaccine formulation can be loaded into the cavity of the microcontainers [27]. In the study, the cavity of the microcontainers was coated with the $\mathrm{pH}$ sensitive polymer Eudragit S100. The release of OVA from the cubosomes and microcontainers was first measured for $2 \mathrm{~h}$ in a $\mathrm{pH}$ value corresponding to the $\mathrm{pH}$ of the stomach $(\mathrm{pH} 1.6)$, and here, as expected, no release was observed due to the intact layer of the Eudragit lid (Fig. 7). After $2 \mathrm{~h}$, the $\mathrm{pH}$ of the medium was changed to reflect that of the small intestine ( $\mathrm{pH} \mathrm{6.8).} \mathrm{Fig.} 7$ shows that the release of OVA is occurring, and this indicate that the cubosomes are also released from the microcontainers as these are empty after the release studies (Fig. 6C). The release is appearing in a more controlled fashion than observed from the unconfined powder cubosomes (Fig. 4). The OVA release in pH 6.8 is $44.1 \pm 5.6 \%$ in relation to the amount of OVA in the particles. This is comparable to the release from the bulk powder being $47.9 \%$ after $96 \mathrm{~h}$ ( $p$ value: 0.4311 ).

In the literature, a rice-based oral vaccine has shown to be efficient as a delivery system as it can protect the antigen from enzymes in the stomach [7]. The microcontainers have the same feature and therefore, there is a promise for the microcontainers to work as an oral vaccine system as well. When delivering vaccines by the oral route, the delivery system should be able to present the vaccine formulation to the $M$ cells followed by transport to the immune cells to create a response. It has been shown to be effective to 
keep the vaccine formulation inside a particle for a significant period of time $[10,12]$, securing a slow release. Therefore the slow release that the microcontainers and the cubosomes provide can be a great advantage when delivering vaccines.

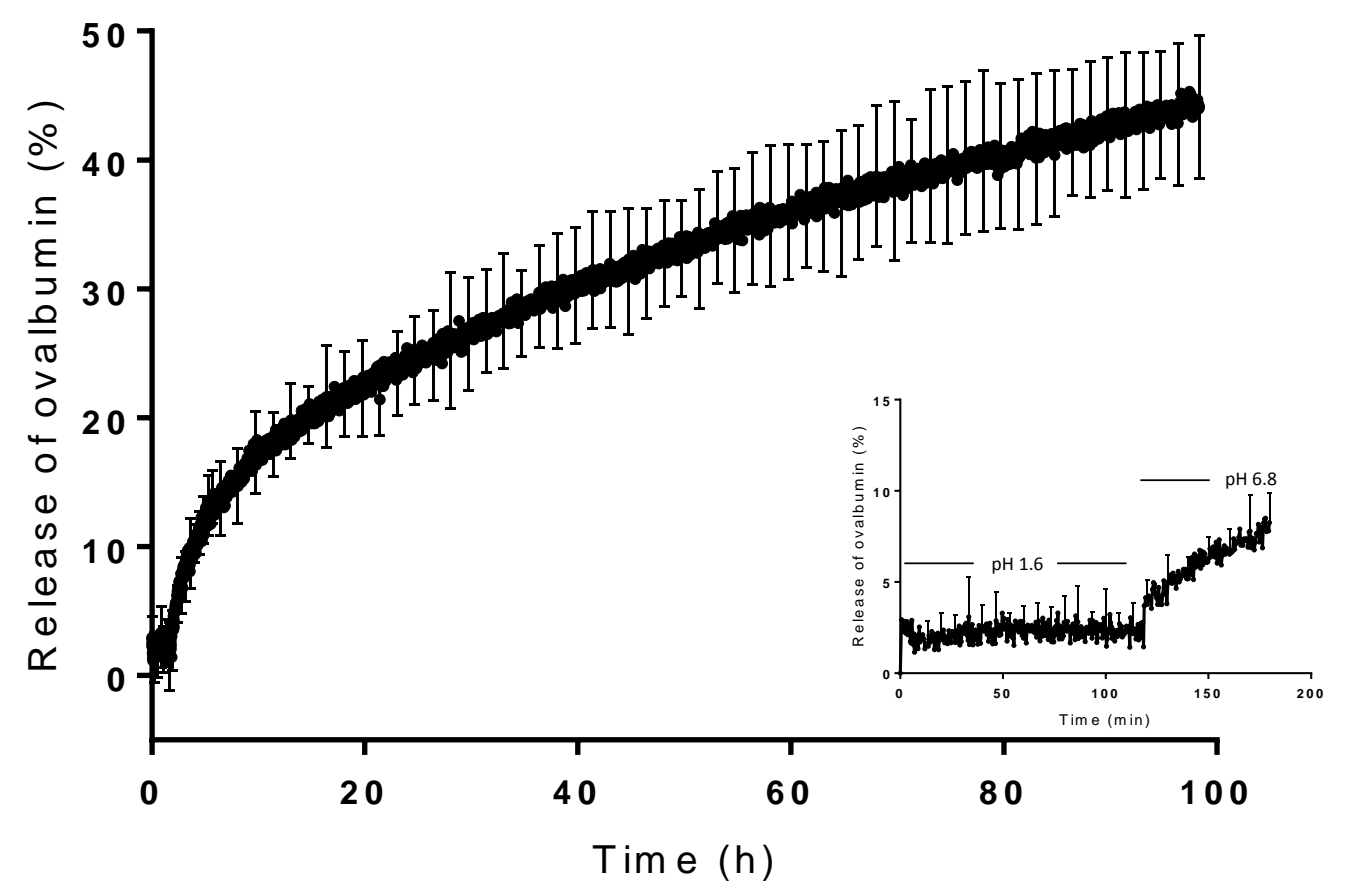

377

Fig. 7: Release of OVA from the cubosomes, when the vaccine formulation was confined in microcontainers. The release of OVA is expressed as a \% of the loaded OVA into the cubosomes. For the first $2 \mathrm{~h}$ the release was measured in $\mathrm{pH} 1.6$ followed by $\mathrm{pH}$ of 6.8 for up to $98 \mathrm{~h}$. The data is presented in triplicates as a mean \pm SD.

\section{Conclusion}

Powder precursors of cubosomes loaded with OVA have been produced by spray drying, and it was concluded that the precursors contained cubic structure in bulk as well as when released from microcontainers. The microcontainers coated with an Eudragit S100 lid can serve as an oral vaccine delivery system protecting the cubosomes through the GI tract until release occurs in the small intestine. For these produced cubosomes to be completely developed as an oral vaccine system, an adjuvant needs to be added to the particles to obtain the optimal effect of this system and further investigations are therefore also needed for fully develop this oral vaccine delivery system.

\section{Acknowledgement}

Assoc. Prof. Stephan Sylvest Keller is acknowledged for the fabrication of the SU-8 microcontainers, and the SAXS/WAXS beamline at the Australian Synchrotron is thanked as parts of these studies were performed there. Furthermore, the authors would like to thank the Danish Research Council for Technology and Production (FTP), Project DFF-4004-00120B for financial support, and in addition, the Danmarks 
397 Grundforskningsfonds (project DNRF122) and Villum Fondens Center for Intelligent Drug Delivery and 398 Sensing Using Microcontainers and Nanomechanics (IDUN) is acknowledged. 
400 [1] Z. Zhao, K.W. Leong, Controlled delivery of antigens and adjuvants in vaccine development, Journal of Pharmaceutical Sciences. 85 (1996) 1261-1270. doi:10.1021/js9602812.

[2] A.M. Stern, H. Markel, The History Of Vaccines And Immunization: Familiar Patterns, New Challenges, Health Affairs. 24 (2005) 611-621. doi:10.1377/hlthaff.24.3.611.

[3] C. Czerkinsky, J. Holmgren, Vaccines against enteric infections for the developing world, Philosophical Transactions of the Royal Society B: Biological Sciences. 370 (2015) 2-13. doi:10.1098/rstb.2015.0142.

[4] A. Gebril, M. Alsaadi, R. Acevedo, A.B. Mullen, V.A. Ferro, Optimizing efficacy of mucosal vaccines., Expert Review of Vaccines. 11 (2012) 1139-55. doi:10.1586/erv.12.81.

[5] X.M. Chen, I. Elisia, D.D. Kitts, Defining conditions for the co-culture of Caco-2 and HT29-MTX cells using Taguchi design, Journal of Pharmacological and Toxicological Methods. 61 (2010) 334-342. doi:10.1016/j.vascn.2010.02.004.

[6] A. Azizi, A. Kumar, F. Diaz-Mitoma, J. Mestecky, Enhancing Oral Vaccine Potency by Targeting Intestinal M Cells, PLoS Pathogens. 6 (2010) e1001147. doi:10.1371/journal.ppat.1001147.

[7] T. Nochi, H. Takagi, Y. Yuki, L. Yang, T. Masumura, M. Mejima, et al., Rice-based mucosal vaccine as a global strategy for cold-chain- and needle-free vaccination., Proceedings of the National Academy of Sciences of the United States of America. 104 (2007) 10986-10991. doi:10.1073/pnas.0703766104.

[8] M. Yu, M. Vajdy, Mucosal HIV transmission and vaccination strategies through oral compared with vaginal and rectal routes., Expert Opinion on Biological Therapy. 10 (2010) 1181-95. doi:10.1517/14712598.2010.496776.

[9] E. Sjögren, B. Abrahamsson, P. Augustijns, D. Becker, M.B. Bolger, M. Brewster, et al., In vivo methods for drug absorption - comparative physiologies, model selection, correlations with in vitro methods (IVIVC), and applications for formulation/API/excipient characterization including food effects., 2014. doi:10.1016/j.ejps.2014.02.010.

[10] R.W. Ellis, Technologies for the design, discovery, formulation and administration of vaccines, Vaccine. 19 (2001) 2681-2687. doi:10.1016/S0264-410X(00)00504-1.

[11] S.B. Rizwan, W.T. McBurney, K. Young, T. Hanley, B.J. Boyd, T. Rades, et al., Cubosomes containing the adjuvants imiquimod and monophosphoryl lipid $A$ stimulate robust cellular and humoral immune responses, Journal of Controlled Release. 165 (2013) 16-21. doi:10.1016/j.jconrel.2012.10.020.

[12] T. Storni, T.M. Kündig, G. Senti, P. Johansen, Immunity in response to particulate antigen-delivery systems, Advanced Drug Delivery Reviews. 57 (2005) 333-355. doi:10.1016/j.addr.2004.09.008.

[13] A. Bolhassani, S. Safaiyan, S. Rafati, Improvement of different vaccine delivery systems for cancer therapy., Molecular Cancer. 10 (2011) 3. doi:10.1186/1476-4598-10-3.

[14] Y. Fujkuyama, D. Tokuhara, K. Kataoka, R.S. Gilbert, J.R. McGhee, Y. Yuki, et al., Novel vaccine development strategies for inducing mucosal immunity., Expert Review of Vaccines. 11 (2012) 36779. doi:10.1586/erv.11.196.

[15] N. Mishra, A.K. Goyal, S. Tiwari, R. Paliwal, S.R. Paliwal, B. Vaidya, et al., Recent advances in mucosal delivery of vaccines: role of mucoadhesive/biodegradable polymeric carriers., Expert Opinion on Therapeutic Patents. 20 (2010) 661-79. doi:10.1517/13543771003730425.

[16] A. Lancelot, T. Sierra, J.L. Serrano, Nanostructured liquid-crystalline particles for drug delivery., Expert Opinion on Drug Delivery. 11 (2014) 547-64. doi:10.1517/17425247.2014.884556.

[17] W. Leesajakul, M. Nakano, A. Taniguchi, T. Handa, Interaction of cubosomes with plasma components resulting in the destabilization of cubosomes in plasma, Colloids and Surfaces B: Biointerfaces. 34 (2004) 253-258. doi:10.1016/j.colsurfb.2004.01.010.

[18] S.B. Rizwan, D. Assmus, A. Boehnke, T. Hanley, B.J. Boyd, T. Rades, et al., Preparation of phytantriol cubosomes by solvent precursor dilution for the delivery of protein vaccines, European Journal of Pharmaceutics and Biopharmaceutics. 79 (2011) 15-22. doi:10.1016/j.ejpb.2010.12.034. 
[19] S. Rizwan, T. Hanley, B. Boyd, T. Rades, S. Hook, Liquid Crystalline Systems of Phytantriol and Glyceryl Monooleate Containing a Hydrophilic Protein: Characterisation, Swelling and Release Kinetics, Journal of Pharmaceutical Sciences. 98 (2009) 4191-4204. doi:10.1002/jps.

[20] J. Kim, H. Kim, H. Chung, Y. Sohn, I. Kwon, S. Jeong, Drug formulations that form a dispersed cubic phase when mixed with water, Rel. Bioact. Mater. 27 (2000) 1118-1119.

[21] P.T. Spicer, K.L. Hayden, M.L. Lynch, A. Ofori-Boateng, J.L. Burns, Novel process for producing cubic liquid crystalline nanoparticles (cubosomes), Langmuir. 17 (2001) 5748-5756. doi:10.1021/la010161w.

[22] P.T. Spicer, W.B. Small, M.L. Lynch, J.L. Burns, Dry powder precursors of cubic liquid crystalline nanoparticles (cubosomes), Journal of Nanoparticle Research. 4 (2002) 297-311. doi:10.1023/A:1021184216308.

[23] A.M. Avachat, S.S. Parpani, Formulation and development of bicontinuous nanostructured liquid crystalline particles of efavirenz, Colloids and Surfaces B: Biointerfaces. 126 (2015) 87-97. doi:10.1016/j.colsurfb.2014.12.014.

[24] M.A. Islam, J. Firdous, Y.J. Choi, C.H. Yun, C.S. Cho, Design and application of chitosan microspheres as oral and nasal vaccine carriers: An updated review, International Journal of Nanomedicine. 7 (2012) 6077-6093. doi:10.2147/IJN.S38330.

[25] L.H. Nielsen, S.S. Keller, A. Boisen, A. Mullertz, T. Rades, A slow cooling rate of indomethacin melt spatially confined in microcontainers increases the physical stability of the amorphous drug without influencing its biorelevant dissolution behaviour, Drug Deliv. and Transl. Res. 4 (2014) 7.

[26] L.H. Nielsen, S.S. Keller, K.C. Gordon, A. Boisen, T. Rades, A. Mullertz, Spatial confinement can lead to increased stability of amorphous indomethacin, European Journal of Pharmaceutics and Biopharmaceutics. 81 (2012) 418-425. doi:10.1016/j.ejpb.2012.03.017.

[27] L.H. Nielsen, A. Melero, S.S. Keller, J. Jacobsen, T. Garrigues, T. Rades, et al., Polymeric microcontainers improve oral bioavailability of furosemide, International Journal of Pharmaceutics. 504 (2016) 98-109. doi:10.1016/j.jpharm.2016.03.050.

[28] L.M. Ensign, R. Cone, J. Hanes, Oral drug delivery with polymeric nanoparticles: The gastrointestinal mucus barriers, Advanced Drug Delivery Reviews. 64 (2012) 557-570. doi:10.1016/j.addr.2011.12.009.

[29] H.D. Chirra, L. Shao, N. Ciaccio, C.B. Fox, J.M. Wade, A. Ma, et al., Planar microdevices for enhanced in vivo retention and oral bioavailability of poorly permeable drugs., Advanced Healthcare Materials. 3 (2014) 1648-54. doi:10.1002/adhm.201300676.

[30] C.L. Randall, T.G. Leong, N. Bassik, D.H. Gracias, 3D lithographically fabricated nanoliter containers for drug delivery, Advanced Drug Delivery Reviews. 59 (2007) 1547-1561. doi:10.1016/j.addr.2007.08.024.

[31] A. Ahmed, C. Bonner, T.A. Desai, Bioadhesive microdevices with multiple reservoirs: a new platform for oral drug delivery, Journal of Controlled Release. 81 (2002) 291-306. doi:10.1016/s01683659(02)00074-3.

[32] S.L. Tao, T.A. Desai, Aligned arrays of biodegradable poly(epsilon-caprolactone) nanowires and nanofibers by template synthesis, Nano Letters. 7 (2007) 1463-1468. doi:10.1021/nl0700346.

[33] L.H. Nielsen, J. Nagstrup, S. Gordon, S.S. Keller, J. Østergaard, T. Rades, et al., pH-triggered drug release from biodegradable microwells for oral drug delivery, Biomedical Microdevices. 17 (2015) 17. doi:10.1007/s10544-015-9958-5.

[34] R.S. Petersen, S.S. Keller, A. Boisen, Hot punching of high-aspect-ratio 3D polymeric microstructures for drug delivery., Lab on a Chip. 15 (2015) 2576-9. doi:10.1039/c5lc00372e.

[35] S. Keller, D. Haefliger, A. Boisen, Optimized plasma-deposited fluorocarbon coating for dry release and passivation of thin SU-8 cantilevers, Journal of Vacuum Science \& Technology B: Microelectronics and Nanometer Structures. 25 (2007) 1903. doi:10.1116/1.2806960.

[36] S.S. Keller, F.G. Bosco, A. Boisen, Ferromagnetic shadow mask for spray coating of polymer patterns, Microelectronic Engineering. 110 (2013) 427-431. doi:10.1016/j.mee.2013.03.029. 
[37] N.M. Kirby, S.T. Mudie, A.M. Hawley, D.J. Cookson, H.D.T. Mertens, N. Cowieson, et al., A lowbackground-intensity focusing small-angle X-ray scattering undulator beamline, Journal of Applied Crystallography. 46 (2013) 1670-1680. doi:10.1107/S002188981302774X.

[38] A.A. Ambike, K.R. Mahadik, A. Paradkar, Stability study of amorphous valdecoxib, International Journal of Pharmaceutics. 282 (2004) 151-162. doi:10.1016/j.ijpharm.2004.06.009.

[39] C.J. Drummond, C. Fong, Surfactant self-assembly objects as novel drug delivery vehicles, Current Opinion in Colloid and Interface Science. 4 (1999) 449-456. doi:10.1016/S1359-0294(00)00020-0.

[40] M.F. Bachmann, G.T. Jennings, Vaccine delivery: a matter of size, geometry, kinetics and molecular patterns., Nature Reviews. Immunology. 10 (2010) 787-96. doi:10.1038/nri2868.

[41] M. Hori, H. Onishi, Y. Machida, Evaluation of Eudragit-coated chitosan microparticles as an oral immune delivery system, International Journal of Pharmaceutics. 297 (2005) 223-234. doi:10.1016/j.ijpharm.2005.04.008.

[42] J. Myschik, F. Eberhardt, T. Rades, S. Hook, Immunostimulatory biodegradable implants containing the adjuvant Quil-A - Part I: Physicochemical characterisation, Journal of Drug Targeting. 16 (2008) 213-223. doi:10.1080/10611860701848860.

[43] H. Aomori, T. Ishiguro, K. Kuwata, T. Kaneko, K. Ogino, Study on thermal and structural behavior of monoacylglycerol-water systems. II. The phase behavior of monooleoylglycerol-water systems., Journal of Japan Oil Chemists' Society. 44 (1995) 1004-1011. doi:10.1017/CBO9781107415324.004.

[44] H. Qiu, M. Ca, The phase diagram of the monoolein / water system : metastability and equilibrium aspects, Biomaterials. 21 (2000) 223-234. 